\title{
Macrocerebellum: Neuroimaging and Clinical Features of a Newly Recognized Condition
}

\author{
John B. Bodensteiner, MD; G. Bradley Schaefer, MD; Gina M. Keller; \\ James N. Thompson, PhD; Mary K. Bowen, MD
}

\begin{abstract}
Other than hamartomatous enlargement of the cerebellum as in Lhermitte-Duclos syndrome, diffuse enlargement of the cerebellum is not clearly described. We report four patients (ages 9 months to 2 years) with diffusely enlarged cerebelli as identified by measurement of the cerebellum and comparison to age appropriate normal values. The cerebellar measurements were determined in absolute numbers and expressed as ratios of cerebellum to whole brain and supratentorial brain. The clinical features of these four children ( 3 boys, 1 girl) consistently include global developmental delay, tone abnormalities, preserved reflexes, delayed or abnormal maturation of the visual system (oculomotor apraxia), and deficient or delayed myelination of cerebral white matter. The etiology of the macrocerebellum is unknown but we propose that the cerebellum is responding to the elaboration of growth factors intended to augment the slow development of cerebral structures. Regardless of the etiology, the finding of a macrocerebellum appears to allow the clinician to predict the clinical features of the patient and probably represents a marker for disturbed cerebral development. ( $J$ Child Neurol 1997;12:365-368).
\end{abstract}

Prior to the development of magnetic resonance imaging (MRI) and more recently, the application of quantitative methods to the analysis of these images, antemortem identification of abnormalities in relative size of various components of the brain was not possible. We have been involved in the morphometric evaluation of MRI scans of children to establish age appropriate normal values for the size and growth of the various brain structures and the range of normal variation of these domains. ${ }^{1-3}$ Our first efforts involved two-dimensional analysis of MRI scans. More recently we have established age appropriate normal values for volumetric measurements of various brain components.

During the 5 years between 1990 and 1994 we identified four children who had abnormally large cerebelli. The

Received Feb 16, 1996. Received revised July 5, 1996. Accepted for publication July 5, 1996.

From the Department of Neurology and Pediatrics, West Virginia University (Dr Bodensteiner), Morgantown, WV, the Department of Pediatrics, Meyer Rehabilitation Institute (Dr Schaefer and Ms Keller), University of Nebraska, Omaha, NE, the Department of Zoology, University of Oklahoma (Dr Thompson), Norman, $\mathrm{OK}$, and the Midlands Hospital (Dr Bowen), Bellevue, NE.

Address correspondence to Dr John B. Bodensteiner, Department of Neurology, West Virginia University Health Sciences Center, Morgantown, WV 26506-9180. clinical features of these children are remarkably similar and constitute a clinically recognizable syndrome. Other than the syndrome of hamartomatous enlargement of the cerebellum (Lhermitte-Duclos syndrome) diffuse enlargement of this structure has not been clearly described as a clinical or pathologic entity. ${ }^{4}$ We report the quantitative neuroimaging findings and clinical features of this newly recognized condition.

\section{PATIENTS AND METHODS}

All patients were initially identified by subjective evaluation of routine MRI scans done as part of clinical evaluations in genetics clinics at the University of Nebraska or neurology clinics at West Virginia University over a 5-year period (1990-1994). Over 1000 patients were evaluated in each clinic each year during this time period. Four patients were found to have abnormally large cerebelli, confirmed by objective two-dimensional morphometric measurement of the MRI or computerized volumetric analysis of the MRI, or both.

MRI scans were obtained on patients seen for clinical evaluations in our respective clinics. Measurements were performed on $\mathrm{T}_{1}$-weighted (repetition time, 500 to $600 \mathrm{~ms}$; echo time 20 to $30 \mathrm{~ms}$ ) images using any number of scanning protocols for each patient with slice width ranging from 3.0 to $5.0 \mathrm{~mm}$ and interslice gap 
from 0.0 to $0.05 \mathrm{~mm}$. Measurements were performed on sagittal series images.

Two-dimensional analysis of the relative size of the cerebellar vermis was done using a computerized image analysis technique by which relative cerebellar area was determined from the midsagittal image and expressed as a ratio of the cerebellar vermis area to the supratentorial brain area and the whole brain area. ${ }^{1,2}$ Quantification of cross sectional areas on individual slices was determined on digitized images on a PC-based system. Tracings and area calculations were made using commercial image analysis software (Image Pro Plus; Media Cybernetics, Silver Spring, MD). Volume was calculated using our own customized algorithm that incorporates slice thickness, interslice gap size, number of slices, and the internal calibration standard. Volumes were expressed as absolute values and as ratios of cerebellum to whole brain and supratentorial brain. These volumes were then compared to 35 agematched controls chosen from a group of scans of normal individuals. The degree of myelination in the cerebral white matter was determined by comparison of the initial MRI scans to the maturational norms for myelination in infants and young children established by Squires et al. ${ }^{5}$

\section{Illustrative Case Report}

Patient 1 was the term product of a gestation to a primigravid mother. The pregnancy was complicated only by a urinary tract infection in the second trimester, requiring antibiotic therapy. Delivery was induced on the expected date of confinement because of maternal hypertension and was accomplished vaginally without complications. He weighed $3.8 \mathrm{~kg}$ and was $52 \mathrm{~cm}$ long with a reported "average" head circumference. He was evaluated at 13 months of age because of developmental delays and was found to have profound central hypotonia with normal reflexes, mild truncal ataxia, and oculomotor apraxia. Growth parameters were at the 95th percentile for age and his head circumference was at the 60 th percentile. His only dysmorphic features were hypoplastic toe nails and a single pigmented macule of the left leg. The diagnostic evaluation included neurometabolic screening, prometaphase chromosomes, and MRI scan of the head.

\section{RESULTS}

All four patients were felt to have large cerebelli on subjective analysis of the MRI scans (Figure 1A and B). However, only three of the four (patients 1, 3, and 4) had abnormally large cerebellar vermi on the two-dimensional analysis of the midsagittal cut of the MRI scan comparing the area of the vermis to the area of the whole brain (Table 1). All patients had cerebellar volumes that were above normal (greater than the mean $+2 \mathrm{SD}$ for the 35 normal controls). The whole brain and supratentorial brain volumes and corpus callosum area were small but within the broad normal range for all patients. Precise age-matched standards do not exist for relative gray- and white-matter volumes but subjective evaluation of the relative size of these components was felt to be normal. The clinical features of the patients with this condition are remarkably similar from one patient to another and generally include global developmental delay, marked early
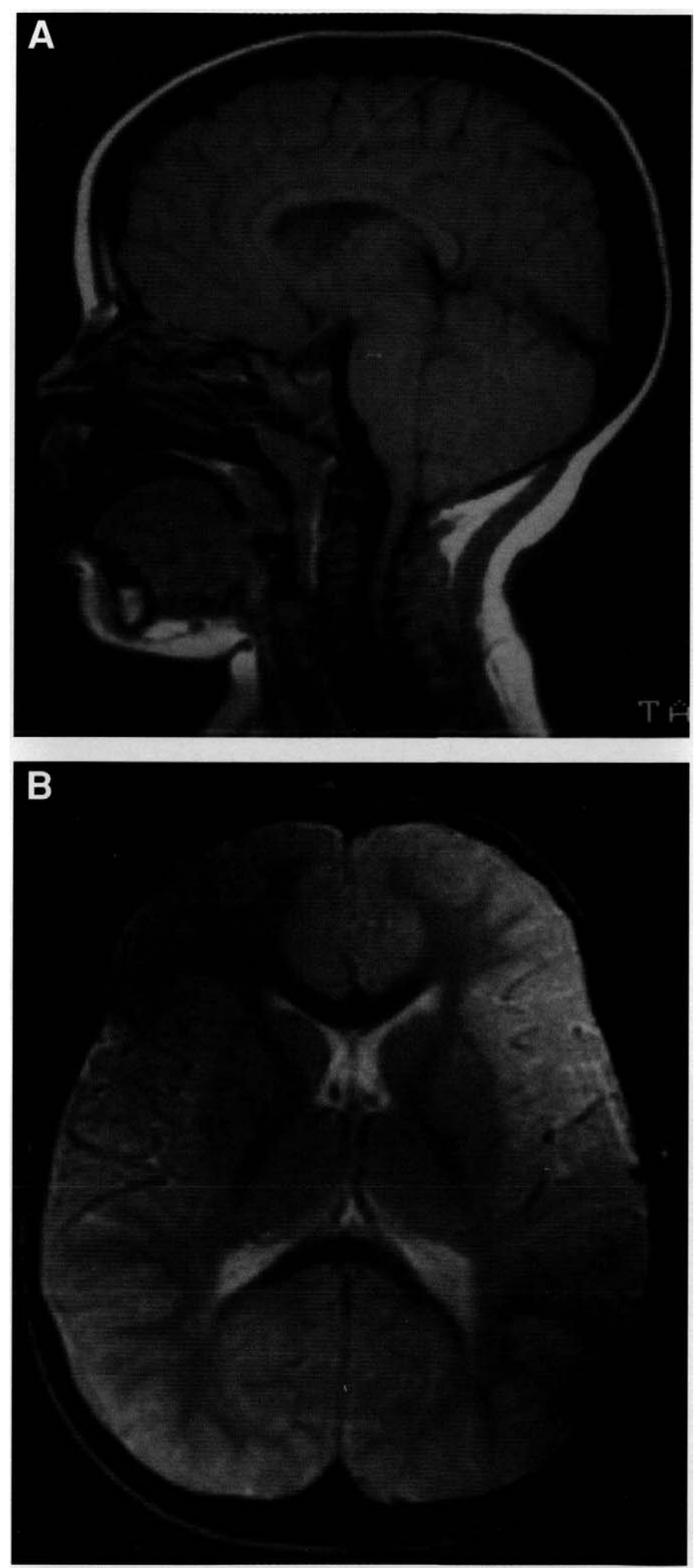

Figure 1. A, Midsagittal cut of $\mathrm{T}_{1}$-weighted cranial MRI of patient 1 showing the diffuse enlargement of the cerebellar vermis. Area and volumetric measurements are used for the determination of macrocerebellum. $B$, Axial cut of $\mathrm{T}_{2}$-weighted cranial MRI of patient 1 showing the widespread myelin deficient cerebral white matter for age.

hypotonia, delayed maturation of the visual system manifested by apraxia of gaze, and delayed maturation of fixation and following skills (Table 2). Ataxia was not a prominent feature of the clinical picture (at least not at this time) 
Table 1. Measurements of Cerebelli

\begin{tabular}{|c|c|c|c|}
\hline & CB $W B$ Area & CBNB Volume & CB/STB Volume \\
\hline Patient 1 & .171 & .121 & .142 \\
\hline Patient 2 & $.083^{*}$ & .133 & .138 \\
\hline Patient 3 & .128 & .137 & .163 \\
\hline Patient 4 & .137 & .137 & .177 \\
\hline Mean of Patients & $.130 \pm 0.031$ & $.132 \pm 0.007$ & $.155 \pm 0.016$ \\
\hline $\begin{array}{l}(n=4) \\
\text { Mean of Controls } \\
(n=35)\end{array}$ & $.0113 \pm 0.07$ & $\begin{array}{c}P<.001 \\
.094 \pm 0.01\end{array}$ & $\begin{array}{c}P<.001^{\top} \\
.112 \pm 0.01\end{array}$ \\
\hline $\begin{array}{l}\text { Mean of Controls } \\
+2 \text { SD }\end{array}$ & .127 & .114 & .132 \\
\hline \multicolumn{4}{|c|}{$\begin{array}{l}\text { *Note that Patient } 2 \text { had a large normal cerebellar vermis area and clearly large } \\
\text { cerebellar volumes. } \\
\text { ' } P \text { values are from a } 2 \text {-tail } t \text {-test comparison of the controls versus our patients } \\
(\mathrm{df}=37 \text { ). } \\
\mathrm{CB}=\text { cerebellum; } W B=\text { whole brain; } \mathrm{STB}=\text { supratentorial brain. }\end{array}$} \\
\hline
\end{tabular}

because the motor incoordination observed was deemed to be consistent with the degree of developmental delay.

All patients had delayed myelination of the cerebral white matter: cerebellar white matter seemed normal though no precise standards exist for determination of the normal range (Figure 1B). One patient had a subsequent MRI (patient 2 ) at 3 years of age and although the degree and distribution of myelin deposition appeared to have improved, the clinical manifestations displayed by this patient did not change.

\section{DISCUSSION}

The clinical features of the patients described here include striking tone abnormalities, delayed motor and cognitive development, mild nonspecific dysmorphic features, absence of pathologic reflexes, and oculomotor apraxia. The chief neuroimaging features are the disproportionately large cerebelli and delayed myelination of the cerebral white matter.

There are no descriptions of isolated disproportionately large cerebelli in the literature. Hamartomatous enlargement of the cerebellum as in the Lhermitte-Duclos syndrome has been reported. Usually the involvement of the

Table 2. Clinical Features of Patients with Macrocerebelli

\begin{tabular}{lcccc}
\hline Feature & Patient 1 & Patient 2 & Patient 3 & Patient 4 \\
\hline Age/Sex & $2.5 \mathrm{yr} / \mathrm{M}$ & $2 \mathrm{yr} / \mathrm{F}$ & $11 \mathrm{mo} / \mathrm{M}$ & $9 \mathrm{mo} / \mathrm{M}$ \\
Motor Delay & Moderate & Severe & Mild & Moderate \\
Cognitive Delay & Moderate & Severe & Moderate & Severe \\
Tone & Decreased & Variable & Decreased & Decreased \\
Reflexes & Normal & Increased & Normal & Normal \\
Coordination & Slightly & Slightly & Slightly & Normal \\
& Decreased & Decreased & Decreased & \\
Ocular Apraxia & Present & Present & Unknown & Present \\
Dysmorphic & Mild & Moderate & Mild & None \\
Myelination & Decreased & Decreased & Decreased & Decreased \\
Follow-up MRI & - & Improved & - & - \\
Head & Normal & Decreased & Decreased & Normal \\
Circumference & & & & \\
\hline
\end{tabular}

cerebellum is focal in this syndrome; however, reports of diffuse enlargement exist. ${ }^{4}$ The imaging and clinical features of the Lhermitte-Duclos syndrome are distinct from the clinical and neuroimaging features shared by these children with macrocerebelli.

Hemi-enlargement, or enlargement of one half of the cerebellum, is recognized in several of the conditions associated with somatic hemihypertrophy including Beckwith syndrome, Russell-Silver syndrome, and Klippel-TrénaunayWeber syndrome. ${ }^{6}$ Each of these conditions has distinctive clinical features that do not generally include striking hypotonia, oculomotor apraxia, or delayed myelination of the cerebral white matter. Furthermore, the cerebellar hemihypertrophy, when it occurs, is usually associated with cerebral hemihypertrophy in these conditions.

Diffuse hypertrophy of the cerebellum with equally severe hypertrophy of the cerebrum manifested as megalencephaly and hydrocephalus has been seen in association with juvenile Alexander disease in one patient. ${ }^{7}$ A single case report of midline hypertrophy of the cerebellum and fornical system in a patient with a meningomyelocele and Chiari II malformation exists. ${ }^{8}$ Once again, the features of these two apparently unique cases do not resemble those of our patients.

The patients reported here all have cerebellar volumes above the normal range. Three of the four also had a significantly increased relative area of the cerebellar vermis. When we compared our patients to an age-matched control group of 35 normal children, the volumetric comparisons were strikingly different $(P<.001)$. Interestingly, the comparison of the areas of the vermi were not statistically different. This suggests that either the vermis is differentially affected in this process or there is a greater variability in its normal size. This is not necessarily surprising as we have already demonstrated that the cerebellar vermis is asymmetrically affected by a variety of neurogenetic processes. ${ }^{9}$ We have also seen two additional patients with large cerebellar vermi and cerebellar volumes at the upper end of the normal range but not beyond two standard deviations. Both of these patients had delayed myelination and shared the clinical features of our macrocerebellum patients. The distinction between these patients and those with a macrocerebellum, if any, is not clear at this time. One possible difficulty is that although our normal range for vermis area is well defined, because our normal population is small in number, the normal range for the cerebellar volumes is not as well established.

The chief neuroimaging feature, in the patients with macrocerebelli, is that the cerebellum is disproportionately large, both absolutely and relatively. The cerebellum appears to have normal neuroimaging features from the standpoint of tissue characteristics. A second uniform neuroimaging feature is that there is delayed myelination of the white matter throughout the cerebrum that is not manifest in the cerebellum. Thus the cerebellum seems to be more mature, from the standpoint of myelin deposition, and perhaps growth, than the cerebrum in these infants. This leads us to 
the hypothesis that the cerebellar tissue is responding in a normal fashion to growth factors elaborated in excess in an attempt to augment the growth and maturation of the cerebrum which is not, for some unknown reason, responding appropriately to these signals. In such a condition, the abnormality would be in the ability of the cerebrum to respond and not intrinsic to the cerebellum. If this hypothesis is correct, the cerebellar enlargement would represent an epiphenomenon not a primary manifestation. Thus one might expect, as in our patients, that the functional abnormalities would primarily be the result of cerebral not cerebellar dysfunction.

We have described four patients with remarkably consistent clinical and neuroimaging features and have chosen to identify them as possessing a macrocerebellum. The clinical features of the macrocerebellum syndrome consist of hypotonia, delayed motor and cognitive development, and oculomotor apraxia. Delayed maturation of cerebral myelin and abnormally large cerebellar size (area and volume) represent the cardinal neuroimaging features of children with macrocerebelli. The pathogenesis of this syndrome is unclear at this time; however, the finding of a macrocerebellum is predictive of cerebral dysfunction in the patient.

\section{References}

1. Schaefer GB, Thompson JN, Bodensteiner JB, et al: Quantitative morphometric analysis of brain growth using magnetic resonance imaging. J Child Neurol 1990;5:127-130.

2. Schaefer GB, Thompson JN, Bodensteiner JB, et al: Age-related changes in the relative growth of the posterior fossa. $J$ Child Neurol 1991;6:15-19.

3. Schaefer B, Bodensteiner J: Growth related changes in the posterior fossa (letter). J Child Neurol 1991;6:372.

4. Milbouw G, Born JD, Martin D, et al: Clinical and radiological aspects of dysplastic gangliocytoma (Lhermitte-Duclos disease): A report of two cases with review of the literature. Neurosurgery 1988;22:124-128.

5. Squires LA, Krishnamoorthy KS, Natowicz MR: Delayed myelination in infants and young children: Radiographic and clinical correlates. J Child Neurol 1995;10:100-104.

6. Anlar B, Yalaz K, Erzen C: Klippel-Trenaunay-Weber syndrome: A case with cerebral and cerebellar hemihypertrophy. Neuroradiology 1988;30:360.

7. Torreman M, Smit LME, van der Valk P, Valk J, Scheltens P: A case of macrocephaly, hydrocephalus, megacerebellum, white matter abnormalities and Rosenthal fibers. Dev Med Child Neurol 1993; 35:732-736.

8. Friede RL, Briner J: Midline hyperplasia with malformation of the fornical system. Neurology 1978;28:1302-1305.

9. Schaefer GB, Thompson JN, Bodensteiner JB, et al: Hypoplasia of the cerebellar vermis in neurogenetic syndromes. Ann Neurol 1996;39:382-385.

\title{
Twenty-Third Annual Meeting of the Southern Pediatric Neurology Society
}

\author{
February 28, 1998, Dallas, Texas
}

For the first time in the history of the Southern Pediatric Neurology Society, the meeting place of the society has been changed from New Orleans to Dallas. This change was made for this year only so as to be affiliated with the CarrellKrusen annual meeting on neuromuscular diseases which will meet in the 2 days preceding our meeting.

The Southern Pediatric Neurology Society meeting will be composed of two segments. One part will represent presentations of the general membership and senior guests. The other segment is reserved for those in training and those enrolled as junior members in the society. All of those in the latter category must be sponsored by a member of the society or by an accredited training director in Pediatrics, Neurology, or Pediatric Neurology. The best paper given at the annual meeting by a person in training will receive the Maggie Duncan Scholarship Award, which represents an honorarium of $\$ 250$. All such persons-in-training presenting papers also automatically become non-dues-paying, junior members of the society.

Abstracts from members of the affiliate neurosciences are also encouraged.

Deadline for receiving abstracts is January 1, 1998 for the meeting of February 28, 1998.

Written abstracts should follow a standard sequence to include introduction, methods and materials, results and conclusions. Yet they should be limited to one double spaced page using standard cpi. The one-page submission should start with the complete title, a list of the consecutive authors (last name and then first name only), and location of the authors in the same sequence as the list of authors with no repetitions. The mailing address and complete telephone number of the communicating author only should be inserted at the bottom of the page. Additionally, the names of known members of the society should be underlined.

General abstracts will be accepted for either oral presentation or poster. All accepted junior category abstracts require oral presentations. Address all abstracts to:

Paul R. Dyken, MD, P.O. Box 70191, Mobile, AL 36670-0191. Fax (334) 476-8277; Tel. (334) 478-6424.

The edited texts of all the accepted abstracts will be published in the Journal of Child Neurology. 